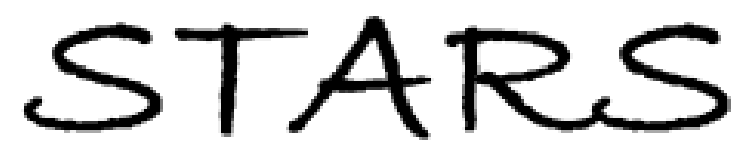

University of Central Florida

STARS

$1-1-1993$

\title{
High-Resolution Photoelectron Spectrometric Analysis Of The Decay Of The 4P Excitations In Atomic Strontium
}

\author{
J. Jiménez-Mier \\ C. D. Caldwell \\ University of Central Florida \\ M. G. Flemming \\ University of Central Florida
}

S. B. Whitfield

P. Van der Meulen

Find similar works at: https://stars.library.ucf.edu/facultybib1990

University of Central Florida Libraries http://library.ucf.edu

This Article is brought to you for free and open access by the Faculty Bibliography at STARS. It has been accepted for inclusion in Faculty Bibliography 1990s by an authorized administrator of STARS. For more information, please contactSTARS@ucf.edu.

\section{Recommended Citation}

Jiménez-Mier, J.; Caldwell, C. D.; Flemming, M. G.; Whitfield, S. B.; and Van der Meulen, P., "High-Resolution Photoelectron Spectrometric Analysis Of The Decay Of The 4P Excitations In Atomic Strontium" (1993).

Faculty Bibliography 1990s. 737.

https://stars.library.ucf.edu/facultybib1990/737

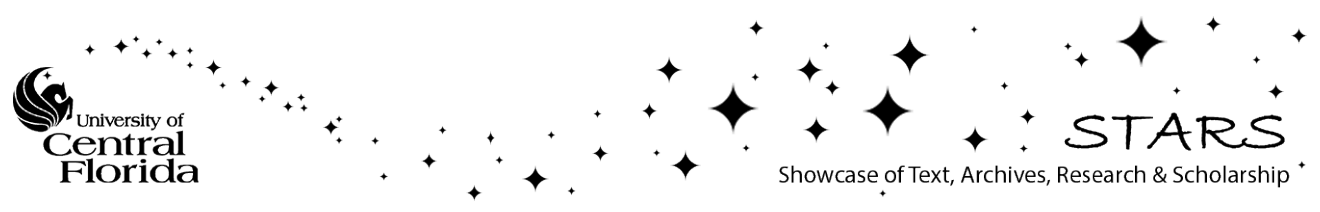




\title{
High-resolution photoelectron spectrometric analysis of the decay of the $4 p$ excitations in atomic strontium
}

\author{
J. Jiménez-Mier \\ Instituto de Ciencias Nucleares, Universidad Nacional Autónoma de México, Apartado Postal 70-453, \\ 04510 Mexico, Distrito Federal, Mexico \\ C.D. Caldwell and M.G. Flemming \\ Department of Physics, University of Central Florida, Orlando, Florida 32816-2385 \\ S.B. Whitfield \\ Fritz-Haber-Institut der Max-Planck-Gesellschaft, Faradayweg 4-6, D-1000 Berlin 33, Germany \\ P. van der Meulen \\ Department of Physical Chemistry, University of Amsterdam, 1018 WS Amsterdam, The Netherlands
}

(Received 10 February 1993)

\begin{abstract}
In a high-resolution analysis of the electron spectrum we have followed the decay of the excitations from the $4 p$ level of atomic strontium. We show that the results of earlier measurements at lower resolution can be accounted for by overlapping resonances. We also find that excitations at higher energy tend to produce highly excited states of the ion, although the production of the states lying lowest in energy remains considerable. Finally, by a comparison with a fluorescence spectrum from the $5 p$ level, we demonstrate that much of the strength associated with the $5 p$ state occurs through cascading from higher levels when they are produced. This behavior severely complicates the interpretation of alignment of excited states when these states are produced by decay of resonances.
\end{abstract}

PACS number(s): $32.80 . \mathrm{Fb}, 32.80 . \mathrm{Hd}$

\section{INTRODUCTION}

Even though the alkaline-earth elements calcium, strontium, and barium have a rather simple electronic configuration, the presence of an often highly polarizable core and an unoccupied $d$ orbital lying just above the $n s^{2}$ outer shell gives rise to considerable configuration mixing. As a consequence of this, the $(n-1) p$ inner-shell absorption spectra of these elements demonstrate a rich multiplet structure [1-5] corresponding to absorption to a complex group of configuration-mixed excited states. The subsequent decay of these absorption resonances by electron emission can involve the participation of a number of electrons. As the decay dynamics of resonance excitations essentially samples the degree of overlap between the wave function of the excited state with that of the final state of the ion which is reached by the decay, resonance electron spectrometry provides an invaluable probe of the details of wave functions for both these species.

In the analysis of resonance absorption in the alkalineearth elements by photoelectron spectrometry, the proper interpretation of the decay through isolation into the final decay channels depends critically on the resolution, both in the exciting photon beam and in the electron analyzer. Photon excitation which overlaps several resonance features will result in a decay distribution which is the incoherent sum of the contributions from the decay paths appropriate to each of the excited states reached by the photon absorption. These might be radically different for each. If one is to avoid obscuring the role of any one decay through overlap with the others, then every attempt must be made to perform the spectrometry measurements at a resolution which will allow maximum isolation of the different excited states.

To date measurements of decay of $(n-1) p$ absorption resonances have been performed for calcium [6], strontium [7], and barium [8]. For both strontium and barium the resolution was not sufficient to adequately resolve the overlapping excitations to such an extent that the details of the decay of each could be isolated. In light of this fact, we have revisited the resonance decay of strontium at a resolution which does allow us to isolate a large number of single features and to reduce others to an overlap of no more than two excited states. In addition to a detailed examination of the features lying lowest in energy, we have also resolved the decay of some of the higher-lying absorption features into the various final-state channels. As a final note, we have compared the decay of these resonances to a spectrum obtained from the fluorescence decay of the $\mathrm{Sr}^{+}\left(4 p^{6} 5 p\right)$ final state [9] in order to isolate the direct contribution to the fluorescence signal from the $5 p$ state alone from that due to cascading of higher-lying states populated in the electron decay of the resonance.

\section{EXPERIMENT}

The bulk of the experiments were performed at the University of Wisconsin Synchrotron Radiation Center 
using the 4-m Normal Incidence Monochromator (NIM). This instrument is capable of wavelength resolution better than $0.02 \AA[10]$. For the strontium experiments discussed in this work, the instrument was used with a bandpass of $0.18 \AA$. As the bandpass of the instrument is constant in wavelength, the equivalent width in energy units varies across the spectrum. In our case the bandpass in wavelength amounted to an energy bandwidth from 5.3 to $6.3 \mathrm{meV}$ in the $25-30 \mathrm{eV}$ region which was of greatest interest. All experiments were made at the "magic angle" so as to avoid any influence on the intensities from unknown asymmetries in the angular distributions of the decay electrons. The strontium was produced in a resistively heated internal oven which has been described earlier [11]. The oven was operated at approximately $500^{\circ} \mathrm{C}$, producing a pressure on the order of 1 mtorr. The decay electrons were analyzed in a spherical sector plate analyzer which has a nominal energy resolution of $1 \%[12]$.

During the course of the analysis, we worked in both the constant-ionic-state (CIS) and photoelectronspectroscopy (PES) modes. The former enabled us first to locate specific resonances, and second to follow the production of specific final states through all the excitation channels. Because it is dominated by the strongest decays, weaker decay channels may not always be identifiable in the total absorption spectrum. However, when isolated in emission through a CIS scan, these weaker decay paths may emerge as the dominant mode of decay of a specific excited state. At the same time, the bandwidth of the electron analyzer, insofar as it is narrow enough to isolate a single final ionic state, does not contribute to the resolution of a CIS spectrum, thus enhancing the overall resolution of the measurement for a specific ionic state. Once a resonance has been isolated through a CIS measurement, the PES measurement then serves to determine the relative intensity distribution of the decay into all the final states which can be observed.

Our measurements were made over an energy region from $20-30 \mathrm{eV}$, although the most detailed analyses were conducted in the $25-29-\mathrm{eV}$ range, in which the strongest excitations occur. All these energies lie above the threshold for double ionization, $16.72 \mathrm{eV}$, but we made no attempt to observe the specific production of the doubly or singly ionized species [13]. We also did not examine the decay channels corresponding to production of excited ionic states of the form $4 p^{5} n \ell n^{\prime} \ell^{\prime}$ [7]. Rather, we concentrated on the resonance decay into the ionic states having a filled core and one outer electron, $\mathrm{Sr}^{+}\left(4 p^{6} n \ell\right)$. Although we recorded a few PES spectra at higher energies, our most detailed analyses were performed in the region below $27 \mathrm{eV}$, where most of the decay typically occurs to the seven ionic states of lowest energy, $5 s, 4 d, 5 p, 6 s, 5 d, 6 p$, and $4 f$. Decay to these final states was investigated through CIS spectra. Decays to final ionic states higher in energy, particularly $6 d, 7 d$, and $8 d$, were observed in the PES spectra but were not followed in detail through CIS scans because of lack of time. We did follow the production of $4 p^{-1}$ hole states above $27.606 \mathrm{eV}$ photon energy through the Auger decay from the limits "4" and "9," $4 p^{5} 4 d\left({ }^{3} P\right) 5 s\left({ }^{2} P_{3 / 2}\right)$ and $4 p^{5} 5 s^{2}\left({ }^{2} P_{3 / 2}\right)$, re- spectively, designated by Mansfield and Newsom [2], with CIS spectra, but this energy region was not analyzed in greater detail by way of PES measurements.

\section{DECAY MODES VIA ELECTRON EMISSION}

The absorption spectrum of atomic strontium in the energy region between 15.5 and $41.3 \mathrm{eV}$ has been analyzed in considerable detail by Mansfield and Newsom [2]. They were able to identify 13 Rydberg series ending on various multiplet states corresponding to a hole in the $4 p$ orbital and valence electrons in the $4 d, 5 s$, and $5 p$ orbitals. However, a great number of the lines they observed could not be assigned to a specific Rydberg series, as is to be expected from the large amount of configuration mixing associated with the atom. In most cases they were able to determine, through comparison with Hartree-Fock calculations, at least one configuration contributing to these lines, even though the contribution might be small. Although we are able to identify some of the excitations we observe with lines in the series, most of the lines we observe correspond to those which could not be assigned to a specific series. Because of this fact we have referred our observed excitations to those of these authors through the energies rather than to specific configuration assignments, which are in most cases not complete.

An example of a photoelectron spectrum recorded at $h \nu=26.990 \mathrm{eV}$ is shown in Fig. 1. This spectrum is chosen for illustration because it has the richest mixture of decays to high-energy states of the $\mathrm{Sr}^{+}$ion and provides the best example of the electron resolution we are able to utilize. The experimental data are indicated with filled circles. In addition, the solid line shows a fit to the data based on a Pearson-7 function [14]. All data have been corrected for the transmission of the electron analyzer. The labels in the figure refer to the different ionic states of the form $\mathrm{Sr}^{+}\left(4 p^{6} n \ell\right)$, including two states $A_{1}$

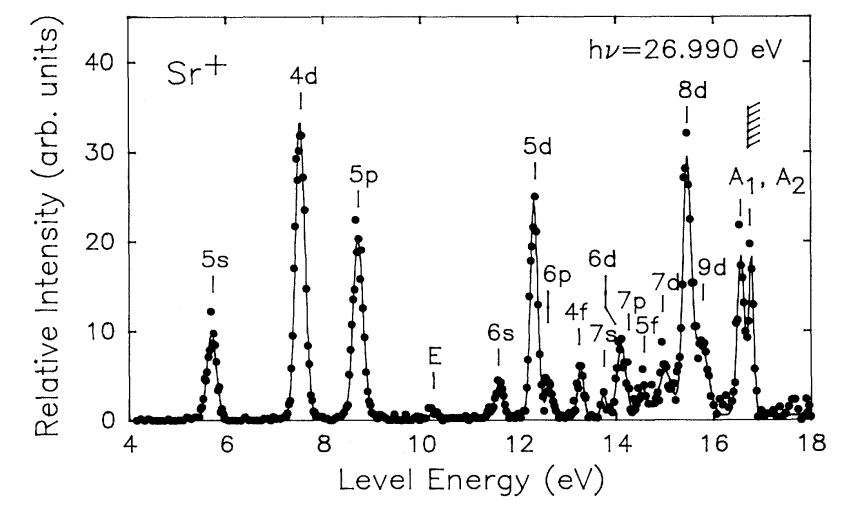

FIG. 1. PES spectrum recorded at $h \nu=26.990 \mathrm{eV}$. Experimental data are given with filled circles; the solid line is a fit to the data. The labels indicate the locations of the final ionic states of the $\mathrm{Sr}^{+}$ion; the double ionization limit is shown at the upper right. Feature $\mathrm{E}$ results from inelastic scattering of electrons out of the $5 s$ state. At the far right are two states, $A_{1}$ and $A_{2}$, which could not be assigned. 
and $A_{2}$ which correspond to levels lying near the ionization limit for which we have no clear assignment. Such features appear only at excitations with the highest photon energy and are characteristic of decays of resonances which lie very near an inner shell ionization threshold [11]. Feature E results from inelastic scattering of electrons corresponding to production of the $5 s$ final states. The location of the $\mathrm{Sr}^{2+}$ ionization limit is given by the hatched lines at the upper right.

The resolution indicated in Fig. 1 enabled us to clearly distinguish the eight final decay channels of lowest energy through production of the $5 s, 4 d, 5 p, 6 s, 5 d, 6 p, 4 f$, and $7 s$ states of $\mathrm{Sr}^{+}$. Production of the $6 d, 5 f, 7 d, 8 d$, and $9 d$ states could also be observed; however, there was some overlap of the $7 p, 8 p, 9 p$, and $10 p$ levels with the $6 d, 7 d, 8 d$, and $9 d$ states due to the resolution of the electron analyzer. In the discussion which follows we will use the designations $n d, n=6-9$ to refer to these states, but it should be understood that contributions from the $n p$ levels, $n=7-10$, may also be present. In our experiments we examined an energy region from 20 to $30 \mathrm{eV}$. For the lowest energies, 20-26.7 eV, the decay occurs predominantly into the first seven states, which are clearly distinguishable in our spectra. Only at higher energies, $h \nu>26.7 \mathrm{eV}$, do the higher-lying $d$ and $p$ states begin to make a significant contribution. Below $25 \mathrm{eV}$ we observed only a few isolated resonances, all of which could be identified on the basis of the work of Mansfield and
Newsom [2]. The principal decay channel of these excitations was into the $4 d$ ionic state. For this reason, and because the most prominent absorption features lie above $25 \mathrm{eV}$, we did not examine this low-energy region further.

In Fig. 2 is shown a summary of the results of our CIS measurements of the resonance decays into the seven final ionic states having the lowest energy. (We do not include the $7 s$ because it is, in general, very weak.) All spectra have been corrected for variations in strontium density and the intensity of the ionizing photon beam. The ordinates of each panel have been appropriately normalized so that the relative intensities can be read directly from the graph. For those channels in which the decay from the excitations of higher energy is weak, $4 s, 4 d, 5 p$, and $5 s$, the upper range of the spectra has been expanded in order to make the patterns visible. Clearly, the decay patterns are very different for the different excitations. In the top left panel are listed the locations of the excited states identified by Mansfield and Newsom [2] from the absorption spectrum. The letters to the left refer to the series which these authors were able to identify; the lines at the bottom could not be assigned to any specific series. The numbers " 4 ," " 9 ," and " 18 " refer to the $4 p^{-1}$ hole states with configurations $4 p^{5} 4 d\left({ }^{3} P\right) 5 s\left({ }^{2} P_{3 / 2}\right)$, $4 p^{5} 5 s^{2}\left({ }^{2} P_{3 / 2}\right)$, and $4 p^{5} 5 s^{2}\left({ }^{2} P_{1 / 2}\right)$, respectively.

In Fig. 3 is given a summed spectrum including contributions from all CIS spectra over the entire range from 25 to $29 \mathrm{eV}$. Between 24.75 and $27.5 \mathrm{eV}$, this spectrum

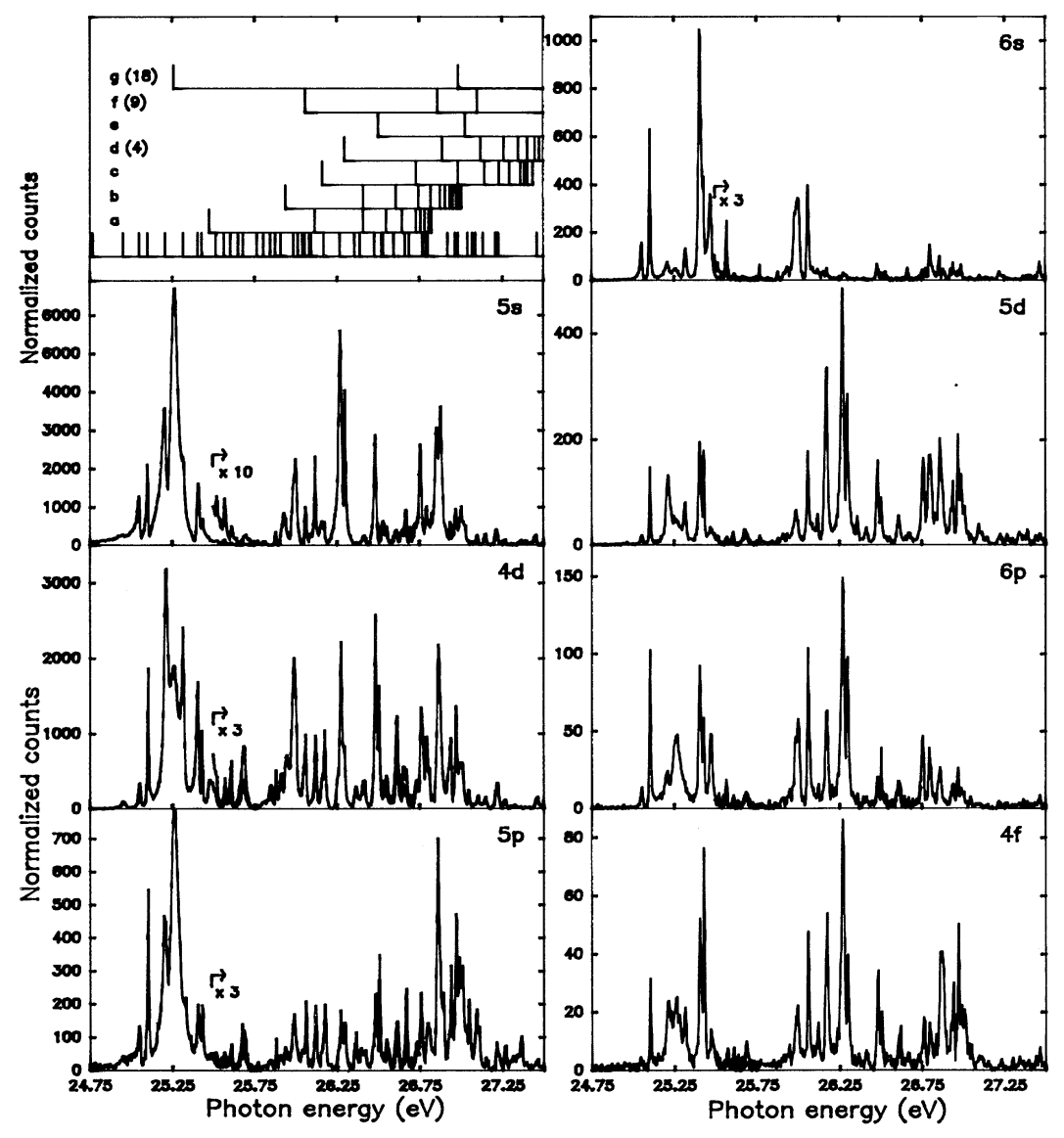

FIG. 2. CIS spectra of the resonances in strontium. Each spectrum is labelled according to the final ionic state examined. The multiplicative factors in the first four spectra multiply the ordinates for the entire energy range from their initial points to 27.5 $\mathrm{eV}$. In the upper left panel is given the location of the excitation features characterized by Mansfield and Newsom [2]. The letters $a-g$ correspond to the series of the same notation which these authors identify; the features on the bottom line are not assigned to a series. 


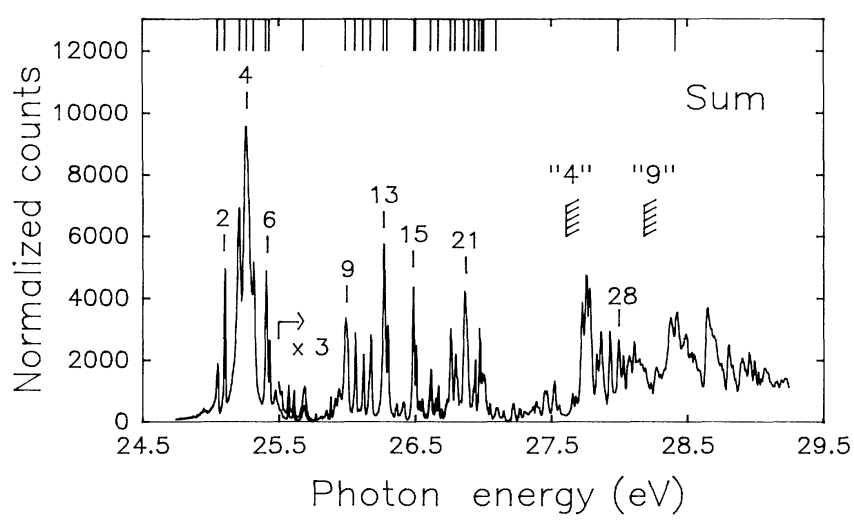

FIG. 3. Summation of all CIS measurements over the region 25-29 eV. Decays into the final states of $\mathrm{Sr}^{+}(5 s, 4 d, 5 p, 6 s, 5 d, 6 p$, and $4 f)$ are included. For the region above $27.5 \mathrm{eV}$ the contributions from the two Auger lines corresponding to the decay of the $4 p$ hole at the limits characterized by " 4 " and " 9 ," shown by hatched lines, are added. At the top of the figure are indicated the energies at which the 29 PES spectra which make up the data in Table I were recorded. The numbers refer to the resonance numbers in Table I.

is a direct summation of all the spectra shown in Fig. 2. Above $27.5 \mathrm{eV}$ are added the contributions from the Auger electrons " 4 " and "9" which result from the decay of the first two $4 p^{-1}$ hole states, shown by hatched lines in the figure, to the ground state of $\mathrm{Sr}^{2+}$. Above the limit "4" the Auger decay vastly predominates over any contribution from the channels $\mathrm{Sr}^{+}\left(4 p^{6} n \ell\right)$. Thus, above this energy, the summed spectrum is essentially that of the Auger contributions alone. While the spectrum in Fig. 3 resembles an absorption spectrum, the relative intensities of the summed peaks do not correspond to the relative intensities of peaks in the absorption spectrum, especially at energies between 26 and $27.6 \mathrm{eV}$, because the summation neglects contributions from decay into channels which were not measured directly, particularly $6 d, 7 d$, and $8 d$. At the top of the figure are given the energy positions of resonance features which were examined in more detail through PES measurements. All resonances are to be identified in numerical order, although some of the more prominent features are specifically labeled.

We determined the energy positions of all the resonance features through the CIS spectra, which were performed in steps of $5-\mathrm{meV}$ photon energy. Thus, the energy of any one spectral feature should be accurate to within $5 \mathrm{meV}$. Because we identify the resonance features through the absorption spectrum obtained by Mansfield and Newsom [2] and because we rely on their calculations to identify the predominant components of these excited states, we have chosen to normalize our energy scale to that of these authors. To do this we converted their wavelength determinations to energy values using the conversion $h \nu(\mathrm{eV})=12398.52 /[\lambda(\AA)]$. Then we used the energy of resonance feature 6 in Fig. 3, given by Mansfield and Newsom as $25.405 \mathrm{eV}$, as the normalization point. We confirmed the assignment of this feature by calculating energy differences between this feature and features $1,2,3,5$, and 7 . All agreed with the corresponding values for these differences listed by these authors to within the 5 -meV step size of our experiment. We do note that we have obtained an energy for resonance 4, the prominent $4 p \rightarrow 4 d$ excitation, which differs from that of these authors by $+9 \mathrm{meV}$. However, this difference could be due to the strong exposure of the photographic plates which this feature produces in the absorption spectrum. In our case we have true relative intensities for this group, features 1-8. Using the energy normalization taken from feature 6 , we determined the energies of the remaining features of our spectrum from our own differences. Each resonance which we observed in detail through PES measurements could be associated with a line listed by Mansfield and Newsom to within an energy less than or equal to the 5-meV step size of our CIS measurements. This gives us confidence that our energy determinations are correct.

Our results for the relative decay distributions at each photon energy we measured are listed in Table I. Because most of our measurements concentrated on the decay into the final states of lowest energy, we have separated out these contributions into individual columns. In order to avoid complexity, we have listed the contributions specifically only for those decay modes in which the branching ratio was greater than 0.01 . The remaining intensity, which for the resonances of lowest energy was too small to be isolated into decay into specific states, is given in the final column. In this column, particularly for the higher photon energies, is also given an indication of where most of the rest of the intensity appears when it is not to be found in the states of lowest energy.

We can make a number of general observations about the relative decay contributions listed in Table I. First, they can be roughly sorted into three energy regions, depending on the relative contributions of the decays to the $5 s$ and $4 d$ final states and the final states of highest energy, $7 d, 8 d, 9 d$, and the unassigned features $A_{1}$ and $A_{2}$. Below $26.7 \mathrm{eV}$ decay to the $5 s$ and $4 d$ states is strong. There is very little contribution from the states above $6 d$, and $5 d$ and $6 d$ are only significantly populated at resonances 12,13 , and 14 . Features 1 and 4 stand out because of a preponderance of decay to the $5 s$ state. Features 8 10 and 15-18 decay primarily to the $4 d$ state. Features 2 , $3,5-7$, and 11 have important decay contributions into both the $5 s$ and $4 d$ states.

The second energy region lies between 26.7 and 27.05 $\mathrm{eV}$. It is characterized by a continuation of the decay into the lower-energy states, $4 d$ in particular, but the contributions from the decays into the states of higher energy, $7 d, 8 d, 9 d$, and $A_{1}$ and $A_{2}$, start to constitute a significant fraction of the total decay. (This is well illustrated in the PES spectrum of Fig. 1.) This behavior is reminiscent of a similar phenomenon in the decay of the $2 p$ excitations in magnesium [11] and the $1 s$ excitations in beryllium [15], although the degree to which the spectrum is dominated by the production of the highly excited states is much less in the case of strontium than 
was true for magnesium and beryllium. For beryllium in particular, decay of all $n p$ excitations into the ground state, $\mathrm{Be}^{+}(2 s)$, was extremely weak. In magnesium, as soon as the resonances of highest energy, $n s, n \geq 6$ and $n d, n \geq 5$, were reached, decay into the $\mathrm{Mg}^{+}(3 s)$ ground state became extremely weak, and the final states having the highest energy became the most prominent decay routes. In strontium, however, we notice that for all resonances up through 24 at $26.975 \mathrm{eV}$ the decay into the $5 s$ and $4 d$ states of the ion amounts to at least $33 \%$ of the sum of all decay channels. Also within this energy region the doubly excited states of the form $4 p^{5} n \ell n^{\prime} \ell^{\prime}$ can be populated above $26.916 \mathrm{eV}$. Their production would generate electrons of much lower kinetic energy than we measured, and we did not attempt to examine these de- cay modes.

We have indicated the two prominent decay features at the high-energy end of Fig. 1 by the notation $A_{1}$ and $A_{2}$. We choose this notation because we are unable to assign these features to any specific final state of the $\mathrm{Sr}^{+}$ion. The corresponding states are higher in energy than any of those listed in the tables by Moore [16], from which we take all our ionic state energies. However, they do lie in energy below the double ionization potential, so the features cannot result from the decay of a $4 p$ hole. In addition, their energies differ with different ionizing photon energy. The appearance of such features is characteristic of decay of excited states of high energy and has been discussed in detail for both magnesium $[11,17]$ and xenon [18]. Through detailed calculations of very accurate wave

TABLE I. Final-state populations produced by decay of selected resonances.

\begin{tabular}{|c|c|c|c|c|c|c|c|c|c|c|c|}
\hline No. & $h \nu(\mathrm{eV})$ & $5 s$ & $4 d$ & $5 p$ & $6 s$ & $5 d$ & $6 p$ & $4 f$ & $7 s$ & $6 d$ & Rest \\
\hline 1 & 25.050 & 0.70 & 0.16 & 0.06 & 0.06 & 0.01 & & & & & 0.01 \\
\hline 2 & 25.105 & 0.37 & 0.34 & 0.10 & 0.11 & 0.03 & 0.02 & & & & 0.03 \\
\hline $3^{a}$ & 25.215 & 0.39 & 0.51 & 0.05 & 0.01 & 0.03 & & & & & 0.01 \\
\hline $4^{\mathrm{a}}$ & 25.265 & 0.70 & 0.20 & 0.08 & & & & & & & 0.02 \\
\hline $5^{\mathrm{a}}$ & 25.315 & 0.46 & 0.43 & 0.04 & 0.02 & 0.02 & & & & & 0.03 \\
\hline 6 & 25.405 & 0.28 & 0.35 & 0.03 & 0.23 & 0.05 & 0.02 & & 0.02 & 0.01 & 0.01 \\
\hline 7 & 25.430 & 0.27 & 0.33 & 0.07 & 0.19 & 0.07 & 0.02 & 0.01 & 0.02 & 0.02 & \\
\hline 8 & 25.680 & 0.07 & 0.72 & 0.11 & & 0.05 & 0.01 & 0.02 & & 0.01 & 0.01 \\
\hline 9 & 25.990 & 0.14 & 0.57 & 0.06 & 0.07 & 0.06 & 0.04 & 0.02 & 0.02 & 0.01 & 0.01 \\
\hline 10 & 26.060 & 0.13 & 0.36 & 0.07 & 0.11 & 0.14 & 0.08 & 0.04 & 0.01 & 0.04 & 0.02 \\
\hline 11 & 26.120 & 0.25 & 0.44 & 0.14 & 0.02 & 0.09 & 0.02 & 0.02 & & 0.02 & \\
\hline 12 & 26.175 & 0.04 & 0.29 & 0.09 & 0.02 & 0.31 & 0.04 & 0.06 & & 0.11 & 0.04 \\
\hline 13 & 26.270 & 0.22 & 0.22 & 0.03 & & 0.22 & 0.05 & 0.04 & & 0.15 & $0.07(5 f, 8 s)$ \\
\hline 14 & 26.295 & 0.26 & 0.17 & 0.05 & & 0.23 & 0.05 & 0.03 & & 0.13 & $0.08(5 f, 8 s, 7 d)$ \\
\hline 15 & 26.485 & 0.18 & 0.50 & 0.06 & 0.01 & 0.12 & 0.01 & 0.03 & 0.04 & 0.02 & 0.03 \\
\hline 16 & 26.505 & 0.03 & 0.49 & 0.12 & 0.01 & 0.12 & 0.02 & 0.03 & 0.04 & 0.06 & 0.08 \\
\hline 17 & 26.615 & 0.06 & 0.61 & 0.08 & & 0.12 & 0.03 & 0.03 & & 0.05 & 0.02 \\
\hline 18 & 26.670 & 0.19 & 0.35 & 0.20 & 0.03 & 0.08 & & 0.01 & & 0.06 & $0.08(0.077 d)$ \\
\hline 19 & 26.760 & 0.18 & 0.32 & 0.05 & & 0.11 & 0.02 & & & 0.09 & $0.23(0.197 d)$ \\
\hline 20 & 26.795 & 0.09 & 0.28 & 0.07 & 0.06 & 0.18 & 0.02 & 0.02 & 0.02 & 0.05 & $0.21(0.167 d)$ \\
\hline 21 & 26.860 & 0.13 & 0.30 & 0.09 & 0.01 & 0.07 & 0.01 & 0.02 & & 0.09 & $0.28(0.127 d ; 0.068 d)$ \\
\hline 22 & 26.895 & 0.25 & 0.23 & 0.11 & & 0.05 & 0.01 & 0.01 & & 0.04 & $0.30(0.177 d)$ \\
\hline 23 & 26.940 & 0.08 & 0.31 & 0.12 & 0.03 & 0.13 & 0.02 & 0.03 & & 0.02 & $0.26(0.158 d)$ \\
\hline 24 & 26.970 & 0.09 & 0.30 & 0.12 & 0.01 & 0.15 & 0.02 & 0.04 & 0.01 & 0.02 & $0.24\left(0.118 d ; 0.08 A_{1}, A_{2}\right)^{\mathrm{b}}$ \\
\hline 25 & 26.990 & 0.06 & 0.21 & 0.13 & 0.02 & 0.11 & 0.01 & 0.02 & 0.01 & 0.04 & $0.39\left(0.148 d ; 0.13 A_{1}, A_{2}\right)^{\mathrm{b}}$ \\
\hline 26 & 27.005 & 0.13 & 0.29 & 0.16 & & 0.08 & 0.01 & 0.03 & & 0.02 & $0.28\left(0.089 d ; 0.12 A_{1}, A_{2}\right)^{\mathrm{b}}$ \\
\hline 27 & 27.095 & 0.03 & 0.08 & 0.12 & 0.01 & 0.04 & & 0.01 & 0.04 & 0.06 & $0.61\left(0.408 d, 9 d ; 0.15 A_{1}\right)^{\mathrm{b}}$ \\
\hline 28 & 27.992 & 0.06 & 0.10 & 0.03 & & 0.06 & & & & 0.03 & $0.72(0.089 \mathrm{~d} ; 0.49 “ 4 ")^{\mathrm{c}}$ \\
\hline 29 & 28.412 & 0.06 & 0.11 & 0.02 & & 0.02 & & & 0.01 & 0.02 & $0.76(0.27 \text { " } 4 " ; 0.40 \text { "9") })^{\mathrm{c}}$ \\
\hline
\end{tabular}

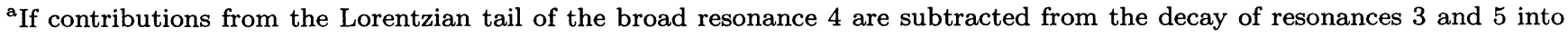
the $5 s$ state, then the relative contributions, $\Gamma(5 s): \Gamma(4 d)$, change to 30:60 and 31:57 for resonances 3 and 5 , respectively. (See text for details.)

${ }^{\mathrm{b}} A_{1}$ and $A_{2}$ refer to decays to unassigned final states near the ionization limit.

c " 4 " and "9" refer to the normal Auger lines resulting from decay of the $5 p$ hole corresponding to the limits of series " 4 " and "9" of Mansfield and Newsom [2]. 
functions, Whitfield, Tulkki, and Åberg [17] were able to show that excitations of very high-lying resonance features primarily decay to states which have higher principal quantum number. Although the calculations were performed in order to reproduce the magnesium decay spectrum, which is not complicated by strong configuration mixing, the fact that these features appear from the decay of more complicated excitations in an atom such as strontium indicates that a sort of "shakeup" to final states having lower binding energy is general for excitations at higher photon energy.

In the third energy region, above $27.05 \mathrm{eV}$, the decay into the states of low energy becomes weak. The production of the final states having highest energy, together with the Auger contributions from the production of the $4 p^{-1}$ hole states above $27.606 \mathrm{eV}$, dominates the spectrum. This is consistent with the observation of Yagishita et al. [7] that, as soon as the $4 p^{-1}$ hole limit " 4 " is reached, most of the absorption results in the creation of this hole, and this is reflected in the preponderance of the intensity of the "4" Auger electron in the spectrum.

An example of the effects of resolution and linewidth on the identification of the decay of an excitation to a group of overlapping resonances is manifest in the decay pattern of the first major resonance seen in the absorption spectrum. This feature is the large group consisting of excitations 3,4 , and 5 in Fig. 3, centered near $25.26 \mathrm{eV}$. Mansfield and Newsom [2] list three states which overlap with these three features, at energies $25.208,25.256$, and $25.316 \mathrm{eV}$, and have assignments of $61 \% 4 p^{5}\left[4 d^{2}\left({ }^{3} P\right)\right] 5 s\left({ }^{3} S\right), 97 \% 4 p^{5} 5 s^{2} 4 d\left({ }^{1} P\right)$, and $35 \%$ $4 p^{5} 4 d\left({ }^{3} P\right) 5 p^{2}\left({ }^{3} P\right)\left({ }^{5} D\right)$, respectively. At our resolution of $5.3 \mathrm{meV}$, we can distinguish between these states in the photon excitation, and we have examined the decay of each of these features separately into the seven decay channels discussed above. In each case roughly $90 \%$ of the decay occurs into the $5 s$ and $4 d$ states. (See Table I.) However, as evidenced in Fig. 2, in which the decays into the final states are shown separately, there are radical differences between the three. These differences are clearly expressed in the numbers given for the individual decay contributions into the $5 s$ and $4 d$ states in the table. However, in the case of these particular excitations, these numbers must be examined more carefully. Resonance 4 , the strong $4 p \rightarrow 4 d$ excitation, is quite broad, as is evident in the CIS spectra for all decay modes (Fig. 2) and in the sum (Fig. 3). In fact, it is the only feature in the absorption spectrum which cannot be completely isolated because of its large width. From a fit of the summed spectrum we determine that this broad feature is well represented by a Lorentzian with a full width at half maximum (FWHM) of $49 \pm 2 \mathrm{meV}$. The effect of this natural width is observable in the decays of features 3 and 5 , as the Lorentzian tail merges with these excitations. Because of the very strong predominance of the decay of feature 4 into the $5 s$ state, the effect of the natural width is to cause an apparent increase in the population of the $5 s$ level at resonances 3 and 5 . Based on the natural width which we determine, we can remove the Lorentzian contribution from resonance 4 to the decay at the energies of resonances 3 and 5 . This results in a change of the numbers given in Table I from $\Gamma(5 s): \Gamma(4 d)=39: 51$ and $46: 43$ to $\Gamma(5 s): \Gamma(4 d)=30: 60$ and $31: 57$ for features 3 and 5 , respectively. These final numbers represent the decay distribution from the specific states corresponding to the energies of 3 and 5 , while the numbers in the table continue to refer to the total decay observable at the resonance.

In their measurements on the decay of the resonance excitations in strontium Yagishita et al. [7] also analyzed this spectral region into contributions from decay into the three states, $5 s, 4 d$, and $5 p$, but they worked with only a $60-\mathrm{meV}$ resolution. From the spectrum which they show in their Fig. 3, they do not distinguish between the three different excitations, but rather measure a sum of contributions from the decays of all three. If we renormalize their results to correspond to a total of 1.00 for the resonant decays alone, neglecting the contribution from the direct decay into $\mathrm{Sr}^{2+}$, we obtain a ratio of populations $5 s: 4 d: 5 p: n \ell$ of $54: 34: 7: 5$. (The contribution $n \ell$ refers to decay into all those states not specifically isolated.) In order to compare our results with theirs, it is necessary to add the relative contributions from the decays of resonance features 3,4 , and 5 , taken from Table I, with the appropriate normalization taken from the CIS spectra, Fig. 2. If we do this, we obtain a ratio of populations $5 s: 4 d: 5 p: n \ell$ equal to $53: 36: 6: 5$. We estimate that our ratios are valid to within $2 \%$. Thus, within the error, our summed results agree very well with those obtained by Yagishita et al. [7].

When the strong $4 p \rightarrow 4 d$ excitation, number 4 in Fig. 2 , is observed with higher resolution, the branching ratios of the decay must be altered. According to the results of Yagishita et al. [7], the ratio, $\Gamma(4 d): \Gamma(5 s)$, corresponding to the decay of this resonance via the two different decay paths, producing $\mathrm{Sr}^{+}(4 d)$ or $\mathrm{Sr}^{+}(5 s)$, is 62:100. However, at the higher resolution, in which resonance 4, corresponding to the dominant $4 p \rightarrow 4 d$ excitation, can be isolated, the branching ratio $\Gamma(4 d): \Gamma(5 s)$ is $29: 100$. This is more in keeping with the result of Bizau et al. [6] for the case of the $3 p \rightarrow 3 d$ excitation in calcium, in which the ratio $\Gamma(3 d): \Gamma(4 s)$ is $10: 100$.

In the general observations on Table I we noted that the excitations 12,13, and 14 constitute somewhat of an anomaly in this energy region in that there is a considerable contribution from the decay into the $5 d$ and $6 d$ states of the ion. While this is not unexpected based on our observations in beryllium [15] and magnesium [11], it falls outside of the pattern of the general behavior of the decays given in the table. Features 12, 13, and 14 correspond to three well-defined lines listed by Mansfield and Newsom [2]. Number 14 corresponds to the excitation in their series to limit " 4 " with configuration $4 p^{5} 4 d\left({ }^{3} P\right) 5 s\left({ }^{2} P_{3 / 2}\right) 5 d$; numbers 12 and 13 are not assigned to a series but are calculated to be $20 \%$ $4 p^{5}\left[4 d\left({ }^{3} D\right)\right]\left(5 p^{2}{ }^{3} P\right)^{1} P$ and $24 \% 4 p^{5}\left[4 d^{2}\left({ }^{1} S\right)\right] 5 s\left({ }^{1} P\right)$, respectively. While one might be tempted to argue that the $5 d$ excitation in feature 14 is demonstrating a type of "shake" behavior in decaying to the $5 d$ and $6 d$ levels, the same cannot be said of features 12 and 13, which have comparable contributions from decay into these states.

If a general pattern for these decays is to emerge, 
then it would most likely be manifest in those resonances which can be assigned to a series. Thus, let us compare the decay of resonance 14 with that of resonances 21 and 22 . Feature 21 corresponds to a level designated by Mansfield and Newsom [2] as part of their series to limit "9" and has configuration $4 p^{5} 5 s^{2}\left({ }^{2} P_{3 / 2}\right) 5 d[3 / 2]_{1}$; feature 22 corresponds to the second component of their series to limit " 4 ," with configuration $4 p^{5} 4 d\left({ }^{3} P\right) 5 s\left({ }^{2} P_{3 / 2}\right) 6 d$. As before, there is a large branching of the decays of these features into the higher-lying states of $\mathrm{Sr}^{+}$, particularly the $7 d$. There is a certain similarity between the decay behavior of resonances 14 and 22 in that the outermost electron appears to be shaken into a higher-lying state. However, there is a considerable difference between the decays of these states into the $5 d$ and $6 d$ levels, respectively. Based on the "spectator" interpretation, there is much more of the $5 d$ "spectator" in resonance 14 than there is of the $6 d$ "spectator" in resonance 22. Moreover, the decay of resonance 21 does not fit into this pattern at all. While it is a $5 d$ excitation, there is little $5 d$ and $6 d$ produced, but a great deal of $7 d$. This argues for a strong influence of the core structure on the decay patterns even in those instances in which one might expect some type of pattern to be visible. This only emphasizes the complicated nature of these excitations and fully demonstrates that it is impossible to draw conclusions about these decays based on models which have applicability to wellisolated single-electron excitations but which clearly do not apply in this case.

Because of the complexity of this spectrum, we have not discussed all decays of all the different excitations which we measured. These are summarized in Table I. For complex atoms with much configuration mixing and often ill-defined orbitals each decay must be examined in isolation, and the various paths do not lend themselves to a simple overall interpretation. Rather, the decay modes serve as a means of efficiently sampling the wave functions of both the excited state and the final ionic state. We have made no attempt to calculate any of these wave functions or to reproduce the theoretical results of Mansfield and Newsom [2]. However, for those instances in which we have a clear resolution into a specific absorption feature, a calculation of the decay distribution could serve as a good check on the accuracy of the wave function above and beyond that of just the reproduction of the energy.

\section{CASCADES IN FLUORESCENCE DECAY}

A complementary mode of study of the process of photoionization is through the analysis of the alignment of the resulting ion. This alignment can be reflected in the linear polarization of radiation from an excited state of the ion if the initial ionization results in the production of an excited state [19]. In a recent experiment Hamdy et al. [9] determined the alignment of the $5 p$ state of $\mathrm{Sr}^{+}$produced through the decay of the various resonant excitations observed in this experiment. The difficulty with the analysis of alignment of an ionic state produced by decay of a resonance is that it is not always clear that the state which fluoresces has been reached through direct decay of the resonance or has been produced by radiative cascading of higher-lying levels which are also produced via the decay of the resonance. This is particularly true for the excitations at higher energy, which tend to decay into higher-lying levels with a probability often much greater than that for the decay into the state being examined.

With this in mind, and with the electron decay spectra which we have obtained in this experiment, we have examined the polarization data obtained by Hamdy et al. [9] with a view toward determining the possible contribution to the fluorescence from radiative cascading from upper states. In so doing we have to consider the possibility both of direct radiative decay into the $5 p$ state and $5 p$ population produced by multiple radiative cascading through one or more intermediate states. In order to determine the relative branching ratios for radiative decay into all allowed channels, we calculated transition probabilities between all of the pertinent $\mathrm{Sr}^{+}$states using wave functions derived from a simple single-configuration Hartree-Fock approximation.

Of those states which are produced, and which are listed in Table I, the $6 s$ and $5 d$ levels can decay by fluorescence emission solely into the $5 p$ state and should, whenever they are produced, make a significant contribution to the fluorescence from the $5 p$ state. The $6 p$ level can decay into all the possible states, $5 d, 6 s, 4 d$, and $5 s$. However, the branching ratios which we calculated for the $6 p$ decay show that the dominant decay mode is into the $6 s$ state (94\%), with a small contribution from decay into the $5 d$ state $(2 \%)$ and the remainder into the $4 d$ and $5 s$ states, which lie below the $5 p$ state in energy. This very strong decay probability for the $6 p$ state into the $6 s$ state has important implications for the cascading process which we are considering. In particular, any higheriying state which decays into the $6 p$ state will make a major contribution to the fluorescence from the $5 p$ state through multiple cascading, first into the $6 p$ state, then from that via the $6 s$ and $5 d$ states into the $5 p$ state. Our branching ratios show this to be the case for the $7 s$ state, $62 \%$ into $5 p$ and $37 \%$ into $6 p$, the $6 d$ state, $67 \%$ into $5 p$, $32 \%$ into $6 p$, the $7 d$ state, $68 \%$ into $5 p$ and $31 \%$ into $6 p$, and so on. The $4 f$ level decays almost exclusively into the $4 d$ state, so it will make no contribution to the fluorescence.

In comparing our results obtained by electron spectrometry with the results of the fluorescence measurements, we have made one major assumption about the relative populations of the final-state levels of highest energy. In particular, we neglect the fact that the $n d$ states, $n \geq 6$, might also have a contribution from the $n p$ states, $n \geq 7$ which is not resolved. We treat these states as if they were $n d$ states alone. We also do not include any contributions from the states of highest energy, designated by the lines $A_{1}$ and $A_{2}$, which we cannot assign. If these states possess a strong $d$ character, they could add significantly to the fluorescence. However, as we have no wave functions with which to calculate branching ratios, we can make no statement about the decay of these states, so we omit them. It is to be understood, when we do this, that our fluorescence spectrum is possibly some- 
what lower in intensity at the highest photon energies because of the neglect of contributions from these states.

While we recognize the fact that our calculation of the population of the $5 p$ state is based on the above assumptions, it is also not our intention to try to reproduce the exact spectrum in every detail. This is also not possible, as there is information missing in the total fluorescence spectrum. In particular, these data were recorded at an angle of $90^{\circ}$ with respect to the quantization axis. The alignment of an excited state produced through photoionization or photoexcitation can be made manifest both in the linear polarization of the decay fluorescence and in the angular distribution of this fluorescence [20]. Thus, the relative heights of the peaks observed in the fluorescence spectrum might be a result of the anisotropic angular distribution of the decay fluorescence and not be solely due to cascading.

The results of our analysis of the fluorescence spectrum under the assumptions discussed above are given in Fig. 4. This figure contains three panels, the uppermost of which corresponds to the fluorescence spectrum measured directly by Hamdy et al. [9] as digitized by us from their Fig. 3. In the lowest panel is given the total electron decay signal determined by us for all states $5 p, 6 s, 6 p, 5 d, 6 d, 7 d, 8 d$, and $9 d$ as discussed in the preceding paragraph. The contributions for the $5 p, 6 s, 5 d$, and $6 p$ states are taken from the CIS scans; the contributions for the $7 s, 6 d, 7 d, 8 d$, and $9 d$ states at the higher energies are taken from the PES spectra outlined in Table I and added to the figure at those specific energies with a bandwidth corresponding to the bandpass of the electron spectrometer. These paths make a considerable contribution in the central region of the spectrum. In the middle panel is given the electron spectrum which we obtain when the high-resolution spectrum in panel (a) is convoluted with a triangular monochromator bandpass of width $40 \mathrm{meV}$ to account for the lower resolution used in the fluorescence measurement. In the lowest panel are also given the locations of the specific resonances observed by us in the PES mode which correspond to specific resonances observed and discussed by Hamdy et al. [9].

As can be seen, there is a good qualitative correspondence between the spectra in panels (b) and (c). For the lower region of the spectrum, up to $25.75 \mathrm{eV}$, the agreement between the two results is very good indeed. For this region, as can be seen from Table I, the principal contribution to the fluorescence spectrum comes from the electron decay to states $5 p, 5 d$, and $6 s$. Thus, our assumptions have little effect on the overall behavior of the curve. Above this energy, we begin to note some differences. Noteworthy, however, is the fact that the two strong features near 26.25 and $26.8 \mathrm{eV}$ appear in both spectra. A direct addition of only those contributions from the $5 p, 5 d, 6 s$, and $6 p$ states is not adequate to account for the strength of these two features. Rather, it is necessary to add the contributions due to cascading from the states of higher energy, particularly $n d, n \geq 6$, to obtain intensities this large, comparable to that due to the strong $4 p \rightarrow 5 d$ absorption near $25.25 \mathrm{eV}$.

Once cascading is taken into account, the polarization measurements no longer reflect solely the alignment of the $5 p$ state but also reflect the alignment, if any, of the states which cascade into this upper fluorescence level. In the strontium spectrum discussed here, the effects of this cascading can be quite severe because, as discussed above, the $5 p$ state is not only populated through direct decay from higher-lying states. Rather, there can be a considerable contribution to the fluorescence from a multiple cascade through the $6 p$ state. This is especially damaging to the polarization measurements because the $6 p$ state decays to the $5 p$ state predominantly through the

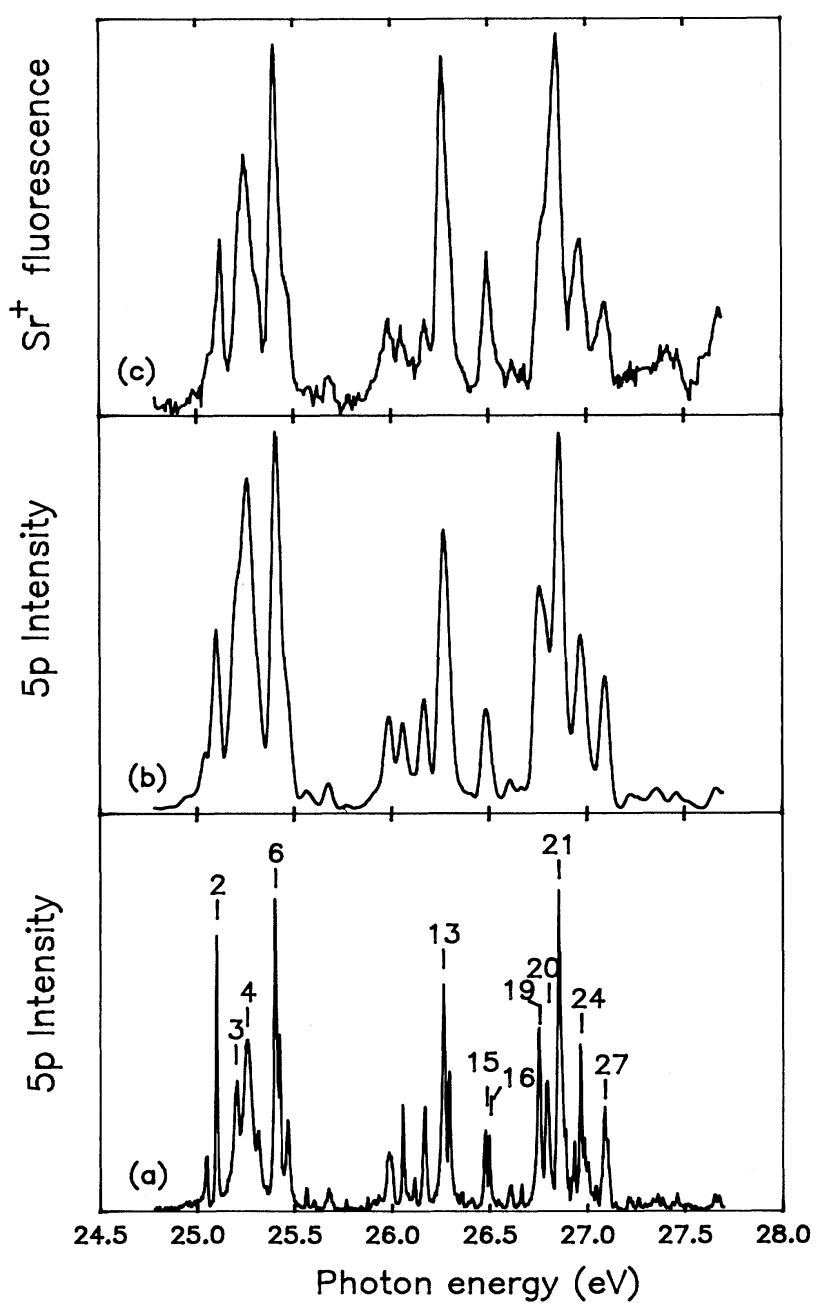

FIG. 4. Fluorescence decay spectrum of $5 p$ state of $\mathrm{Sr}^{+}$. In panel (a) is given the total production of the $5 p$ state as determined through direct production and cascading from higher-lying states derived from our high-resolution electron spectrometry measurements. Panel (b) shows these high resolution results convoluted with a spectral bandpass of $40 \mathrm{meV}$ to correspond to that used in the direct fluorescence measurements. In panel (c) is given the fluorescence spectrum determined by Hamdy et al [9] as digitized from their Fig. 3. The similarity between panels (b) and (c) attests to the fact that much of the fluorescence which these authors are observing derives from cascading to the $5 p$ state from levels lying higher in energy. 
$6 s$ state, which depolarizes the fluorescence completely. Thus, in isolating the possible alignment of a state of high energy, it is necessary to not only consider the population of that state, but also to take into account what percentage of the radiative decay proceeds directly to the $5 p$ state and what percentage proceeds via the $6 p$ state.

In Table II we have separated out the contributions to the polarization at the resonance features at which our PES measurements correspond to those selected by Hamdy et al. [9]. In the first column are the energies given by these authors and in the second column the corresponding resonance taken from our Table I. In the third column are given the values for the linear polarization determined experimentally, but corrected for the polarization of the ionizing radiation, taken from Table 1 of Hamdy et al. In the fourth column is given the percentage contribution to the fluorescence from direct production of the $5 p$ state, based upon a value of 1.00 for the total fluorescence. Only in the case of resonance 4 does this contribution amount to a significant fraction of the fluorescence. The remainder derives from cascading from the $5 d(0.08), 6 s(0.07)$, and $6 p(0.06)$ states. Even though the $6 s$ and $6 p$ contributions act to depolarize the fluorescence, it is clear, because of the large contribution of direct $5 p$ production to the radiative decay, that the $5 p$ state is aligned to some extent. It is also possible that the $5 d$ state could be aligned, resulting in some net contribution to the polarization within the cascade, but this alignment cannot be ascertained on the basis of this one polarization measurement alone. If the $5 d$ state were not aligned at all, the measured value of the linear polarization, -0.133 , based on the total fluorescence intensity, would correspond to an equivalent linear polarization of the fluorescence from the $5 p$ state of -0.168 , derived based on the intensity of fluorescence from the $5 p$ state alone. Cascading from the $6 s, 5 d$, and $6 p$ reduces this number through contributions to the total measured intensity. Because there is a net final polarization which

TABLE II. Fluorescence polarization and direct contribution from $5 p$ population.

\begin{tabular}{cclc}
\hline \hline$h \nu(\mathrm{eV})^{\mathrm{a}}$ & $\begin{array}{c}\text { Our } \\
\text { resonance }\end{array}$ & $\begin{array}{l}\text { Experimental } \\
\text { polarization }^{\mathrm{a}}\end{array}$ & $5 p$ \\
\hline 25.108 & 2 & -0.140 & 0.37 \\
25.231 & 3 & $-0.039^{\mathrm{b}}$ & 0.54 \\
25.246 & 4 & $-0.133^{\mathrm{b}}$ & 0.79 \\
25.262 & 6 & -0.012 & 0.10 \\
25.277 & 13 & -0.205 & 0.06 \\
25.412 & 15,16 & -0.103 & 0.25 \\
26.279 & 19 & -0.112 & 0.11 \\
26.779 & 20 & -0.125 & 0.13 \\
26.802 & 21 & -0.154 & 0.20 \\
26.872 & 24 & +0.003 & 0.25 \\
26.989 & 27 & -0.124 & 0.17 \\
27.107 & \hline
\end{tabular}

${ }^{a}$ Energies and values of the polarization are taken from the measurements of Hamdy et al. [9].

b Since our resonances overlap with two of the experimental values in this case, we use the average value of the polarization. can be derived based solely on the fluorescence intensity from the $5 p$ state, even if all other contributions are not aligned, the conclusion that the $5 p$ state is aligned is certainly correct. In general, however, one must be very careful in drawing conclusions about the alignment of these various states when cascades are involved. For example, although the contributions to the fluorescence from direct production of the $5 p$ state are the same in the overlapping features, 15 and 16, and in feature 24 , the polarization is completely different.

Although the results presented for this fluorescence spectrum as calculated through the contributions from the electron decay spectrum are not entirely quantitative, they do demonstrate that cascading can be a major contributor to fluorescence emission when the latter is measured at a resonance excitation. In order to correctly isolate the alignment of any of the ionic states, it is necessary to make a polarization measurement of the radiative decay of each excited state individually. However, even these measurements must be combined with a resonance decay spectrum to isolate all the different cascades before it becomes possible to extract the alignment of each state alone. Nonetheless, a combination of a resonance decay spectrum with an alignment measurement provides a further check on the accuracy of the wave functions and serves as a strong control over the observation of the electron dynamics underlying the decay.

\section{CONCLUSIONS}

In the foregoing we have presented a detailed highresolution analysis of the decay of the excitations from the $4 p$ level in strontium. We have succeeded in isolating many of the excitations and demonstrating that the subsequent decay of many excitations having very similar character can be very different. Because of the sensitivity of the decay dynamics to the wave functions of both the excited state of the neutral species and the final ionic states to which it decays, this mode of observation should serve as an excellent means of analysis of these wave functions. Although we have not attempted any sophisticated calculations relating to this process, it is clear that an explanation of the results which we observe will require a detailed theoretical analysis which correctly interprets the multielectron character of these wave functions. Finally, the analysis and interpretation of the photoelectron data revealed a strong contribution from cascading into alignment measurements made through fluorescence decay when the decay is observed at a resonance excitation. Proper interpretation of the alignment of the state can only be made through isolation of the contribution from the cascades either by way of combination with electron spectra or with additional fluorescence measurements of the cascades. Detailed decay spectra of those resonances which were not discussed in detail in the manuscript are available upon request.

\section{ACKNOWLEDGMENTS}

This work was supported by the National Science Foundation under Grants Nos. PHY-8907286 and 
INT-9002102. J.J.M. also acknowledges support from CONACYT under Contract No. 6140105-4400-G000-909 and P.v.d.M. from the Netherlands Organization for Scientific Research. We would like to thank M.O. Krause for participation in the analysis and for a critical reading of the manuscript. The Synchrotron Radiation Center is supported by the National Science Foundation under Grant No. DMR-8821625.
[1] M.W.D. Mansfield and G.H. Newsom, Proc. R. Soc. London, Ser. A 357, 77 (1977).

[2] M.W.D. Mansfield and G.H. Newsom, Proc. R. Soc. London, Ser. A 337, 431 (1981).

[3] R.A. Roig, J. Opt. Soc. Am. 66, 1400 (1976).

[4] J.P. Connerade, M.W.D. Mansfield, G.H. Newsom, D.H. Tracy, M.A. Baig, and K. Thimm, Philos. Trans. R. Soc. London 290, 327 (1979).

[5] S.J. Rose, I.P. Grant, and J.P. Connerade, Philos. Trans. R. Soc. London 296, 527 (1980).

[6] J.M. Bizau, P. Gerard, F.J. Wuilleumier, and G. Wendin, Phys. Rev. A 36, 1220 (1987).

[7] A. Yagishita, S. Aksela, Th. Prescher, M. Meyer, M. Richter, E. von Raven, and B. Sonntag, J. Phys. B 21, 945 (1988).

[8] P.H. Kobrin, R.A. Rosenberg, U. Becker, S. Southworth, C.M. Truesdale, D.W. Lindle, G. Thornton, M.G. White, E.D. Poliakoff, and D.A. Shirley, J. Phys. B 16, 4339 (1983).

[9] H. Hamdy, H.-J. Beyer, J.B. West, and H. Kleinpoppen, J. Phys. B 24, 4957 (1991).

[10] C.D. Caldwell and M.O. Krause, J. Phys. B 23, 2233 (1990).
[11] S.B. Whitfield, C.D. Caldwell, and M.O. Krause, Phys. Rev. A 43, 2338 (1991).

[12] M.O. Krause, T.A. Carlson, and A. Fahlman, Phys. Rev. A 30, 1316 (1984).

[13] T. Nagata, J.B. West, T. Hayaishii, Y. Itikawa, Y. Itoh, T. Koizumi, J. Murakami, Y. Sato, H. Shibata, A. Yagishita, and M. Yoshino, J. Phys. B 19, 1281 (1986).

[14] K. Pearson, Biometrika 16, 157 (1924).

[15] C.D. Caldwell, M.G. Flemming, M.O. Krause, P. van der Meulen, C. Pan, and A.F. Starace, Phys. Rev. A 41, 542 (1990).

[16] C.E. Moore, Atomic Energy Levels, Natl. Bur. Stand. (U.S.) Circ. No. 467 (U.S. GPO, Washington, D.C., 1949).

[17] S.B. Whitfield, J. Tulkki, and T. Åberg, Phys. Rev. A 44, 6983 (1991).

[18] S.B. Whitfield, C.D. Caldwell, D.-X. Huang, and M.O. Krause, J. Phys. B 25, 4755 (1992).

[19] C.D. Caldwell and R.N. Zare, Phys. Rev. A 9, 2383 (1977).

[20] J. Jiménez-Mier, M.G. Flèmming, C.D. Caldwell, and D.L. Ederer, Phys. Rev. A 44, 5615 (1991). 